\title{
Fachbeitrag
}

\author{
Matthias Harbeck, Matthias Kaun
}

\section{Voraussetzungen von Nachhaltigkeit: Technische Entwicklungen bei den Fachinformationsdiensten}

http://doi.org/10.1515/abitech-2019-4003

Zusammenfassung: Die Fachinformationsdienste (FID) für die Wissenschaft stehen nach der Evaluation des Förderprogramms vor der Aufgabe, enger zu kooperieren und auf- bzw. miteinander abgestimmte Dienstleistungen bestenfalls gemeinsam zu entwickeln, zumindest aber vorhandene Services und Tools nachzunutzen. Ziel ist es, aus den für sich und ihre jeweilige Fachcommunity weitgehend unabhängig arbeitenden FID ein System zu formen, das zu einer abgestimmten, nachhaltigen Informationsinfrastruktur heranwächst. Grundlage hierfür sind vor allem gemeinsame technische Entwicklungen. Ein Beginn wurde durch die Einrichtung einer Arbeitsgruppe zu technischen Fragen gemacht. Diese Zusammenarbeit muss nun in beständige, transparentere und technisch unterstützte Bahnen gelenkt werden.

Schlüsselwörter: Fachinformationsdienste (FID), Technische Entwicklung, Kooperation

\begin{abstract}
Following the evaluation of the programme, the Specialised Information Services for researchers face the tasks of cooperating more closely and, at best, developing services and tools together in a more coordinated effort. The aim is to refine the Specialised Information Services, which up to this point work mostly individually and solely in the interest of their specific academic communities, and turn it into a system that will grow into a coordinated, sustainable information infrastructure. Joint technical developments are the basis for this transformation. A start has been made by the implementation of a subcommittee on technical issues. This cooperation now needs to be steered onto a more regular and transparent path that is supported by the technical infrastructure.
\end{abstract}

Keywords: Specialised Information Services, Technical Development, Cooperation, Sustainability

\section{Einleitung}

Das DFG-Förderprogramm „Fachinformationsdienste für die Wissenschaft“", ${ }^{1}$ das nach seiner Neuauflage im Jahr 2014 für einige Veränderungen und frischen Wind in der Bibliothekslandschaft sorgte, wurde nun nach fast sechs Jahren Laufzeit einer turnusmäßigen Evaluation unterzogen: Die Evaluierungskommission, die in den Jahren 2017 und 2018 unter Mitwirkung der Firma Prognos mit der Evaluation der FID beauftragt war, hat eine Bestandsaufnahme und Empfehlungen vorgelegt, die einige Veränderungen einleiten bzw. jüngste Entwicklungen verstärkt vorantreiben werden. ${ }^{2}$ Während ein Teil der Empfehlungen organisatorischer Natur ist, betrifft vieles technische Herausforderungen und setzt auf eine Ausweitung und Vertiefung der Kooperationen in diesem Bereich. ${ }^{3}$ Dieser Artikel wird einige Aspekte davon beleuchten und nächste Schritte skizzieren. Es geht insgesamt darum zu zeigen, wie und mit welchen technischen Mitteln die FID zukünftig als nachhaltiges System besser aufgestellt und weiterentwickelt werden können.

Dabei meint Technik hier nicht nur hochkomplexe technische Infrastrukturen wie z.B. virtuelle Forschungsumgebungen oder Forschungsdatenrepositorien mit differenziertem vorgeschalteten Rechtemanagement, sondern bezieht sich auch auf Informationsangebote nach innen

1 Deutsche Forschungsgemeinschaft. „Information zum Förderprogramm ,Fachinformationsdienste für die Wissenschaft‘." https:// www.dfg.de/foerderung/programme/infrastruktur/lis/lis_foerderangebote/fachinfodienste_wissenschaft/ (02.08.2019).

2 Vgl. die entsprechenden Publikationen der DFG: Deutsche Forschungsgemeinschaft. „Weiterentwicklung des Förderprogramms ,Fachinformationsdienste für die Wissenschaft': Stellungnahme der Kommission zur Evaluierung des Förderprogramms ,Fachinformationsdienste für die Wissenschaft"“ https://www.dfg.de/download/pdf/dfg_im_profil/zahlen_fakten/programm_evaluation/ studie_fid_stellungnahme.pdf (07.08.2019); Susanne Heinzelmann u. a. „Evaluierung des Förderprogramms ,Fachinformationsdienste für die Wissenschaft': Studie von Studie von prognos im Auftrag der Deutschen Forschungsgemeinschaft (DFG).“ https://www.dfg.de/ download/pdf/dfg_im_profil/zahlen_fakten/programm_evaluation/ studie_fid.pdf (07.08.2019).

3 Vgl. auch den Aufsatz von Vivien Petras in diesem Heft. 
(zwischen den FID) und außen (zu anderen Infrastruktureinrichtungen), die technisch unterstützt werden können. Bietet man alle Informationen zu den Angeboten und Aktivitäten der FID in einer übersichtlichen, digitalen, durchsuchbaren Form an, gelingt es viel leichter, eine größere Transparenz und Erreichbarkeit herzustellen - ein Zusammenwirken im System wird hierdurch erleichtert. Das von der Staats- und Universitätsbibliothek Hamburg betreute und von den FID kooperativ gepflegte Portal Webis ${ }^{4}$ ist ein erster Schritt in diese Richtung, der bislang aber nur allgemeine Erstinformationen und Einstiege bietet. Hier kann die Informationsqualität zukünftig durch eine verbindlichere Dokumentation und Nutzung maßgeblich verbessert und es können weitaus größere Synergieeffekte erzielt werden.

Eine verbreitete Befürchtung im Hinblick auf die FID ist deren mangelndes systemisches Zusammenwirken und die separat, ohne Austausch stattfindende Erarbeitung von Lösungen für vergleichbare Probleme. Kritiker sehen hier die Gefahr ineffizienter Doppelarbeit analog zu den Erfahrungen beim Aufbau der ersten virtuellen Fachbibliotheken von Sondersammelgebieten (SSG). Während die DFG als Fördereinrichtung grundsätzlich keine Top-downVorgaben für geförderte Entwicklungen verordnet (allerdings in anderen Förderprogrammen mit Praxisregeln und Handreichungen bereits klare Rahmensetzungen vorgenommen hat), wurde durch die Evaluation deutlich, dass hier mehr Koordination und Synergien erreicht werden müssen, um zu einem effizienteren, nachhaltig arbeitenden System zu kommen. Denn ein systematischer Austausch von Arbeitsergebnissen und kooperative Entwicklungen sind nötig, um die maßgeschneiderten Lösungen, die in Folge verbesserter Kommunikation zwischen FID und Fachcommunitys entstanden sind, breiter zu nutzen.

\section{Unterschiedliche Systeme im Bibliothekswesen}

Eine Grundproblematik, die sich aus der zunehmenden Notwendigkeit kooperativ und nachhaltig $\mathrm{zu}$ arbeiten ergibt, liegt in Systemunterschieden bzw. -überschneidungen zu dem übergeordneten System der Bibliotheken in Deutschland, Europa und der Welt einerseits und zu dem lokalen System der jeweiligen FID-Einrichtung ande-

4 AG Fachinformationsdienste. „Webis.“ Staats- und Universitätsbibliothek Hamburg https://wikis.sub.uni-hamburg.de/webis/index. php/Webis_-_Sammelschwerpunkte_an_deutschen_Bibliotheken (02.08.2019). rerseits. Michael Knoche erkennt etwa in seinem Beitrag zur Festschrift für Thomas Bürger eine klare Priorisierung der lokalen Bedürfnisse bei den Hochschulen: „Die Hochschulleitungen sehen sich in ihrem Rollenverständnis den eigenen Hochschulangehörigen stärker verpflichtet als der Allgemeinheit und steuern die Bibliotheken entsprechend. " 5 Die nationalen Aufgaben, die er identifiziert und die in Teilen auch in Verantwortungsbereiche der FID fallen, sieht er als „unerledigt“ an. ${ }^{6}$ Er kommt zu dem Schluss, dass ,aus den vielen guten Bibliotheken [...] noch kein gutes System der Bibliotheken geworden" sei. ${ }^{7}$

Ein Beispiel aus den FID, das veranschaulicht, wo ein Zusammenwirken durch konkurrierende Systeme verhindert wird, sind die in den FID-Portalen verwendeten Content-Management- und Discovery-Systeme: Während Typo3 eines der am häufigsten verwendeten ContentManagement-Systeme (CMS) im deutschsprachigen Raum sowie in der Bibliothekswelt und daher auch bei vielen FID ist, hat die Universitätsbibliothek der Humboldt-Universität zu Berlin das CMS ihres Fachportals EVIFA mit dem Wechsel zum FID-Programm von Typo3 auf Plone umgestellt - und das aus gutem Grund: Zwar hätten theoretisch technische Neuentwicklungen in Typo3 von anderen FIDPortalen nachgenutzt werden können, aber da die Humboldt-Universität zu Berlin insgesamt auf Plone als CMS setzt und entsprechend weniger Expertise für Typo3 vorhanden ist, überwiegen die hausinternen Synergieeffekte bei der Pflege des Portals nach dem Wechsel zu Plone. Die Gewährleistung einer nachhaltigen Betreuung durch das lokale System hat hier Vorrang vor eventuellen - und bis dato noch nicht in größerem Maße erzielten - Synergieeffekten im sich entwickelnden FID-System.

Das Authentifizierungssystem der Staatsbibliothek zu Berlin wurde vor mehr als zehn Jahren im Kontext des SSG Ost- und Südostasien entwickelt und eingeführt und wird heute von der gesamten Einrichtung genutzt. Die Staatsbibliothek zu Berlin hat darüber hinaus in den letzten Jahren entschieden, dass die technischen Plattformen, die ein FID des Hauses erstmals nutzt, von anderen FID nachund mitgenutzt werden müssen. Parallel dazu werden die in den FID erarbeiteten technischen Lösungen ggf. von der Staatsbibliothek zu Berlin im Rahmen ihrer direkten Services genutzt. So basieren die Webangebote aller FID der Staatsbibliothek zu Berlin auf dem CMS Typo3 und der

5 Knoche, Michael. „Was macht die Zusammenarbeit von Bibliotheken so schwierig?" In Kooperative Informationsinfrastrukturen als Chance und Herausforderung. Festschrift für Thomas Bürger zum 65. Geburtstag. Hrsg. von Achim Bonte, Juliane Rehnolt. Berlin: De Gruyter, 2018. 43-52, hier: 44.

6 Knoche 2018: 50-51.

7 Knoche 2018: 51. 
eigenen Plattform von Wordpress. Der Einsatz von Pazpar2 als Discovery-System wird technisch ebenfalls gebündelt betreut. Die seit zwei Jahren aufgebauten und nun im Einsatz befindlichen Fedora-Instanzen als Repositorium, z. B. für das Integrierte Text-Archiv (ITR) für den FID Asien, werden nun auch von den anderen FID und auch von der Staatsbibliothek zu Berlin in ihren sonstigen Kontexten genutzt. Nur so können in den Häusern die innovativen Entwicklungen der FID für ihre Community auch von Nutzen für die übrigen Zielgruppen der FID-Einrichtung sein. Gleichzeitig bietet die Integration der technischen Plattformen und Lösungen der FID-Services in die der Einrichtungen eine größtmögliche Absicherung der Angebote.

Hier überlappen sich also mehrere Systeme, die noch nicht fein aufeinander abgestimmt sind. In Zeiten knapper Ressourcen wird verständlicherweise aus arbeitsökonomischen Erwägungen und mit Blick auf die Nachhaltigkeit im Falle einer Beendigung der Förderung vielerorts auf direkte Nachnutzbarkeit und kurze Kommunikationswege (im Haus bzw. innerhalb eines FID) gesetzt. Die nachhaltigste Strategie besteht für fast alle FID darin, ihre technischen Infrastrukturen mit den Entwicklungen im eigenen Haus zu verzahnen. Für FID, die von mehreren Häusern betrieben werden, gilt das entsprechend für die jeweils entwickelnde Einrichtung. Dass diese Entscheidungen zurzeit überwiegend zugunsten lokaler Lösungen fallen, deutet auf die noch nicht ausreichend etablierte Zusammenarbeit zwischen den FID hin; wirklich gemeinsame Entwicklungen stellen zurzeit noch eine Seltenheit dar. Nichtsdestotrotz werden aber bereits an einem Ort erarbeitete technische Lösungen in anderen FID oder auch im Kompetenzzentrum für Lizenzierung ${ }^{8}$ nachgenutzt. Dies ist z. B. bei der Authentifizierung und Autorisierung für die Nutzung lizenzrechtlich geschützter Inhalte der Fall. ${ }^{9}$ Auch aufgrund des leergefegten Stellenmarkts für IT-Kräfte und der oftmals nur befristeten, vergleichsweise schlecht dotierten Projektstellen in den FID muss über Möglichkeiten der Zusammenarbeit und der Nachnutzung bei der Entwicklung technischer Infrastruktur dringend diskutiert werden: Im Bereich von CMS und Discovery-Systemen, evtl. auch bei der Entwicklung von generischen Repositorien können FID von vorhandenen Expertisen profitieren oder als Innovationsmotoren bzw. Versuchslabore für die sonstigen Services der FID-Einrichtungen fungieren. Es ist z. B. nicht ausgeschlossen, dass der BERT-SolR-Index für EVIFA, das Fachportal Ethnologie zukünftig für den

8 https://www.fid-lizenzen.de/ (12.09.2019).

9 So profitiert z.B. das Kompetenzzentrum für Lizenzierung von der Shibboleth-Lösung des FID Asien der Staatsbibliothek zu Berlin.
Katalog der Humboldt-Universität adaptiert wird. Viele der überregional ausgerichteten Serviceangebote der FID haben aber, mit Ausnahme z.B. der bereits überregional ausgerichteten Staatsbibliotheken, natürlicherweise im lokal orientierten Serviceportfolio der FID-Einrichtungen keine Entsprechung. Hier liegen also Felder, die deutlich als Kooperationsbereiche zu identifizieren sind.

Die technische Umsetzung von standortunabhängigen Verfügbarkeitsanzeigen in den FID-Nachweisinstrumenten ist ein zentraler Baustein der FID-Portale. Es ist letztlich deren Auftrag, den Recherchierenden nicht nur bibliografische Informationen zu liefern, sondern sie nach Möglichkeit zum Suchtreffer zu führen - gleichgültig ob als online zugreifbare E-Ressource oder bestellpflichtiges Material. Bei den sehr heterogenen Daten, die von den FID für die Nachweisinstrumente aus meist sehr unterschiedlichen Datenquellen zusammengesammelt oder geharvestet werden, ist ein umfassender Standortnachweis nicht trivial. Ein Workshop der Unterarbeitsgruppe Technische Infrastrukturen der AG der FID zum Thema „Verfügbarkeitsrecherche“ am 17. Juni 2019 in Hamburg hat hierbei gezeigt, dass die FID unabgestimmt agieren. Der Umstand aber, dass es zu einem solchen Workshop kam, der den Austausch vorantreiben sollte und in dessen Folge sicherlich mehrere der vorgestellten Ansätze von anderen FID nachgenutzt werden, zeigt, dass bereits ein System im Werden begriffen ist. Allerdings muss der Kommunikationsprozess zu solchen Fragen verstärkt organisiert werden, teilweise über die FID hinaus, wie die vielversprechende Implementierung des OpenURL-Gateway-Resolvers ${ }^{10}$ des Hochschulbibliothekszentrum des Landes Nordrhein-Westfalen (hbz) zeigt: Dieses Instrument, das auch verhältnismäßig einfach in die Portale integrierbar wäre, ist dann erfolgreich, wenn eine größtmögliche Anzahl von Einrichtungen - und eben nicht nur die FID-Einrichtungen - sich in die zugrundeliegende Datenbank einträgt und diese Daten auch aktuell hält. Nur dann funktioniert auch für die Nutzenden der Portale die Prüfung, ob ihre eigene Bibliothek oder wenigstens ihr Verbund den im Portal gefundenen Titel vorhält bzw. liefern kann. Sollten sich die FID darauf einigen können, dass dieses Instrument eine ideale Teillösung für die Herausforderung der Verfügbarkeitsrecherche darstellt, könnten die FID-Einrichtungen mit ihrem vereinten Gewicht das bibliothekarische System auffordern, die Datenbank des hbz entsprechend zu pflegen. Ein einzelner FID erreicht hier kaum die gleiche Wirkung. Nichtsdestotrotz kann konstatiert werden, dass FID schon heute nicht abgeschottet agieren, sondern wesentlich stärker als zu SSG-Zeiten in ihre lokalen und vor allem die jeweiligen

10 https://openurlgw.hbz-nrw.de/register (12.09.2019). 
Verbundstrukturen integriert und zuweilen auch Innovationstreiber sind.

Dass eine verbesserte Abstimmung zwischen den FID und ihren Träger-Einrichtungen notwendig ist, wurde im Bereich des Umgangs mit Forschungsdaten relativ früh deutlich: Obwohl in einigen Fächern bereits seit Jahrzehnten oder zumindest Jahren Fragen der Archivierung, Bereitstellung/Nachnutzung und Analyse von Forschungsdaten erörtert und bearbeitet werden, gibt es weite Bereiche der geistes- und kulturwissenschaftlichen Fächer, die hier noch keine etablierten Lösungen vorfinden. Auch an den Universitäten sind meist erst in den vergangenen Jahren generische, nicht-fachspezifische Angebote entwickelt und implementiert worden. Die FID haben sich in diese Entwicklung eingebracht - sei es als Kommunikator und Multiplikator in die Fachcommunitys hinein, sei es als Partner entsprechender Speichereinrichtungen - und sind auch bei Einrichtungen, die Entwicklungstrends nur zurückhaltend aufgreifen, oftmals eine treibende Kraft in der Diskussion um Forschungsdatenmanagement und Forschungsdatenarchivierung. ${ }^{11}$ Mit der durch den Rat für Informationsinfrastrukturen vorgeschlagenen groß angelegten Förderung einer Nationalen Forschungsdateninfrastruktur (NFDI) sind hier nationale - wenn nicht sogar internationale - Arbeitsfelder identifiziert worden, deren Aufbau Top-down organisiert werden soll. ${ }^{12}$ Die FID sind hierbei als denkbare Partnereinrichtungen der sogenannten Konsortien, die wissenschaftsgeleitet diese Struktur organisieren und aufbauen sollen, frühzeitig einbezogen und somit in ein übergeordnetes System integriert worden, das sich bemüht, die verschiedenen Systeme und Ebenen der Forschung und Informationsinfrastruktur zu berücksichtigen und einzubinden.

11 Harbeck, Matthias, Matthias Kaun. „Forschungsdaten und Fachinformationsdienste - eine Bestandsaufnahme.“ Bibliothek Forschung und Praxis 43,1 (2019): 35-41.

12 Informationen zur Ausschreibung findet man hier: Deutsche Forschungsgemeinschaft. „Nationale Forschungsdateninfrastruktur - Ausschreibung 2019 für die Förderung von Konsortien. “ Information für die Wissenschaft 37 (2019). https://www.dfg.de/foerderung/info_wissenschaft/2019/info_wissenschaft_19_37/index.html (07.08.2019). Die AG der FID hat sich früh im Prozess als Partner positioniert, vgl. „Gemeinsame Stellungnahme der FID leitenden Einrichtungen und der AG Fachinformationsdienste (AG FID) zur Einbindung von Fachinformationsdiensten in den Aufbau einer Nationalen Forschungsdateninfrastruktur.“ Arbeitsgemeinschaft der Fachinformationsdienste der Sektion 4 des DBV. https://wikis.sub.uni-hamburg.de/webis/images/a/a1/AG_FID_zu_NFDI.pdf (03.01.2019).

\section{Transparenz und Sichtbarkeit der technischen Entwicklungen}

Wenn Probleme nicht in einer koordinierten übergeordneten Struktur mit institutionalisierten Austauschmechanismen wie in der NFDI verhandelt werden, wird es schwieriger, technische Entwicklungen und Kooperationen transparent zu machen: Im FID-System stellt es eine noch größere Herausforderung dar nachzuvollziehen, welche technischen Entwicklungen wo stattfinden/-fanden und ob ggf. eine Dokumentation der technischen Umsetzung vorzufinden ist. Ohne eine solche Transparenz, die durch einmal im Jahr stattfindende Workshops zu einem Thema oder kurze Inputs auf AG-Sitzungen nicht ausreichend hergestellt werden kann, wird sich keine wirkungsvolle Zusammenarbeit entfalten. Hierfür benötigen die FID eine aktiv genutzte Austausch- und Dokumentationsplattform. Auch hierfür gibt es mit dem bereits im System der Sondersammelgebiete (SSG) etablierten Wiki Webis eine Ausgangsbasis, die derzeit vor allem zur Erstinformation über die FID nach außen und zur Dokumentation der AGSitzungen genutzt wird. Der vorhandene Webis-Blog ${ }^{13}$ müsste stärker und aktiver genutzt werden, um die Entwicklungen nachvollziehbar zu dokumentieren. Selbst die DFG-Geschäftsstelle, die anhand der Anträge alle Informationen vorliegen hätte, konnte erst im Rahmen der Evaluierung eine grobe Zusammenschau der unterschiedlichen FID-Services bieten. Eine Service-Matrix, die die Ziele, Leistungen und den jeweiligen Planungs-/Bearbeitungsstand der einzelnen FID übersichtlich darlegt, ist bislang aufgrund ihrer Komplexität ein Desiderat geblieben.

Ein weiteres Desiderat der FID betrifft Fragen der oben bereits genannten Authentifizierung. Diverse Services der FID - von den registrierungspflichtigen FID-Lizenzen über personalisierte Suchen zu News- und Dokumentlieferservices - erfordern eine Anmeldung und Authentifizierung. Portalnutzende lehnen aber nichts mehr ab, als sich mehrfach irgendwo anmelden und ggf. sogar an unterschiedlichen Stellen verschiedene Registrierungen vornehmen $\mathrm{zu}$ müssen. Die Aufforderung der Evaluationskommission, den Zugang zu Information möglichst einfach, niedrigschwellig und - das ist wiederum eine neue Erkenntnis der Evaluation und ein leichter Paradigmenwechsel im FID-Programm - möglichst breit zu ermöglichen, verstärkt nochmals den Ursprungsgedanken des Förderpro-

13 AG Fachinformationsdienste. „Webis-Blog.“ Staats- und Universitätsbibliothek Hamburg. https://blogs.sub.uni-hamburg.de/webis/ (02.08.2019). 
gramms. ${ }^{14}$ Auch über die Grenzen eines FID hinweg: Es wird hierbei beispielsweise an Soziologinnen gedacht, die auch philosophische Ressourcen nutzen möchten, oder Geologen, die Ressourcen der Kartographie benötigen. Dies muss nicht nur beim Zuschnitt der Lizenzierung und den verhandelten Lizenzmodellen bedacht, sondern auch bei der Registrierung und ggf. Anmeldung in den unterschiedlichen Portalen berücksichtigt werden. Der Einsatz von Shibboleth, wie er bereits seit Jahren im FID Asien an der Staatsbibliothek zu Berlin umgesetzt wird, scheint hier Möglichkeiten zu bieten, ist aber im deutschen Bibliothekssystem nur bedingt flächendeckend einsatzbereit. Gleichwohl sollte nicht vergessen werden, dass FIDLizenzen insofern eine Besonderheit darstellen, als sie „quer“ zu den sonstigen, meist lokalen Lizenzen liegen. Technisch und administrativ ist die Akkumulation von Nutzungsrechten, die sich aus unterschiedlichen institutionellen Zugehörigkeiten ergeben, eben ziemlich komplex. Angesichts einer stärker überregional oder auch international ausgerichteten Wissenschaft und Forschung werden hier mit Sicherheit einige FID Vorreiter einer durchlässigeren Zugangsstruktur sein - eine Chance für die FID, sich hier zu engagieren bei gleichzeitigen Hürden in den lokalen Einrichtungen, die diesen Bedarf ggf. noch nicht adressiert bzw. aufgrund ihrer lokalen und regionalen Ausrichtung diese Aufgabe noch nicht identifiziert haben.

Ähnlich wichtig wäre es, bei Fragen der Archivierung digitaler Angebote (z. B. Webseiten mit oder ohne multimediale oder interaktive Module) kooperativ und auch über die FID hinaus Lösungen zu erarbeiten. Durch die unterschiedlichen Bedarfe von National- und Landesbibliotheken einerseits sowie oftmals international sammelnden FID andererseits und in Ermangelung einer zentralen Koordinierungsstelle sind hier bislang eher zufällige Kooperationen zustande gekommen. Programme wie die im Entstehen begriffene NFDI versuchen, daraus zu lernen, und nehmen schon im Vorfeld eine stärkere Koordinierung vor. Mit der AG Emulation gibt es im Netzwerk nestor eine Anlaufstelle, die aber wenig institutionalisiert und nicht mit Mitteln ausgestattet ist, um diese große Aufgabe wirklich für alle Stakeholder zu lösen. ${ }^{15}$ Die Deutsche Nationalbibliothek hat hier mit dem Projekt „Emu-

14 Deutsche Forschungsgemeinschaft. „Evaluierung und Stellungnahme zum Programm ,Fachinformationsdienste für die Wissenschaft'““ https://www.dfg.de/foerderung/programme/infrastruktur/ lis/lis_foerderangebote/fachinfodienste_wissenschaft/fid/index. html (24. 07.08.2019).

15 nestor. „AG Emulation.“ https://www.dnb.de/Webs/nestor/ DE/Arbeitsgruppen/AG_Emulation/ag_emulation_node.html (07.08.2019). lation im Lesesaal“"16 eine für ihre Bedürfnisse angepasste Lösung erarbeitet, die allerdings den Anforderungen des FID-Förderprogramms nicht gerecht wird (keine überregionale Bereitstellung, keine Archivierung nicht-deutscher Inhalte) und darüber hinaus die drängenden rechtlichen Fragen nicht abschließend beantwortet.

Einen neuen Weg bei der Archivierung von Webseiten geht der FID Zentralasien, indem er über eine amerikanische Partnerorganisation das Internet Archive ${ }^{17}$ mit der Archivierung ohne Zustimmung der Rechteinhaber betraut. Die rechtlichen Implikationen, die das haben könnte (Archivierung findet in den USA statt, daher könnte amerikanisches Recht gelten), sind hierbei in Kauf genommen worden, um das Ziel einer Archivierung nicht-deutschen Materials zu ermöglichen. Inwiefern diese Lösung zukünftig umfassend digitale Angebote für den Zugriff und die Nutzung bereitstellen kann, hängt wiederum von den Entwicklungen beim Internet Archive ab.

Eine Übersicht über die Überlegungen und Anstrengungen im Bereich der Archivierung und Verfügbarmachung von Webangeboten und digitalen, interaktiven Medienformen sowohl innerhalb des FID-Systems als auch national wie international könnte konzertierte Aktionen und gemeinsame Entwicklungen ermöglichen. Diese Mammutaufgabe wird vermutlich keine Einrichtung allein zufriedenstellend stemmen können.

\section{Fazit}

Die angeführten Beispiele illustrieren, dass es bereits innovative technische Entwicklungen im Bereich der FID gibt, diese aber bislang recht isoliert entwickelt wurden und nur vereinzelt eine Nachnutzung durch andere FID gefunden haben. Mit der Gründung der Unter-AG für technische Infrastrukturen hat die AG der FID dieses Desiderat erkannt und sich im Rahmen ihrer Möglichkeiten bemüht, den Prozess der Kooperation voranzutreiben. Flankiert durch die Evaluationsergebnisse werden diese Bemühungen zukünftig verstärkt und durch einen systematischen Ausbau der Organisationsstrukturen unterstützt werden. Es bleibt zu hoffen, dass dies nicht nur durch eine neue Governance-Struktur, die regelmäßig tagende und im Austausch stehende Arbeitsgruppen zu den Bereichen vorsieht, sondern auch durch Kommunikations- und Doku-

16 Steinke, Tobias. „Projekt EMiL - Emulation im Lesesaal.“ Deutsche Nationalbibliothek. https://www.dnb.de/SharedDocs/Downloads/DE/Kulturell/konferenzeDigKultErbe2018_TobiasSteinke. pdf?_blob=publicationFile\&v=2 (07.08.2019).

17 https://archive.org/ (12.09.2019). 
mentationsplattformen erreicht wird, die entsprechende Entwicklungen transparent sichtbar und teilbar machen. Wichtig bei all diesen Aktivitäten ist, dass die FID zuallererst Dienste für ihre Disziplin, ihre Fächer oder Regionen im Zusammenspiel mit Forschung und Wissenschaft, Bibliotheken und anderen Informationseinrichtungen erbringen. Die Nutzung der Angebote, die Akzeptanz seitens der Wissenschaft steht bei allen FID-Aktivitäten im Vordergrund. Die Integration der Services eines FID in die Services und Strukturen der FID-Einrichtung bietet dabei heute die bestmögliche Absicherung der Angebote. Eine deutlich verbesserte Kultur der gegenseitigen Information, des Austauschs und vertrauensvoller Kooperation auch in Bezug auf Service- und Softwareentwicklungen wird diesen Nachhaltigkeitsaspekt verstärken.

\section{Autoreninformationen}

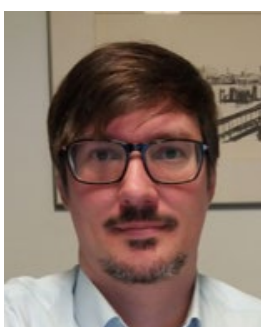

Matthias Harbeck

Universitätsbibliothek der HumboldtUniversität zu Berlin

Unter den Linden 6

10099 Berlin

matthias.harbeck@ub.hu-berlin.de orcid.org/0000-0002-7909-5967

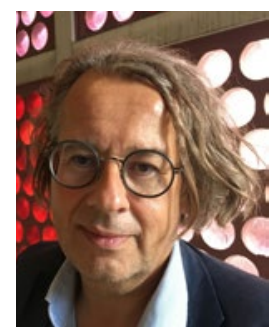

Matthias Kaun

Staatsbibliothek zu Berlin | Ostasienabteilung

Potsdamer Str. 33

10785 Berlin

matthias.kaun@sbb.spk-berlin.de

orcid.org/0000-0002-6528-1830 